\title{
Ureteroscopic lithotripsy; retrospective review of mid and lower ureteric stones- its results and complications
}

\begin{abstract}
Purpose: To observe efficacy and complications of ureteroscopic lithotripsy in the treatment of mid and lower ureteric stones. The placement of DJ Stent was also compared for their beneficial role or otherwise

Materials and Methods: The medical records of 268 patients who underwent ureteroscopic lithotripsy (URSL) by a single surgeon from January 2010 December 2013 were reviewed retrospectively. All adult patients of mid and lower ureteric stones $(10-22 \mathrm{~mm})$ were included in this study. Group I ( $\mathrm{n}=98)$ patients had no DJ stent while Group II $(\mathrm{n}=96)$ patients had DJ stent due to prolong procedure. Group III $(\mathrm{n}=74)$ patients had DJ stent placed due to complications during the procedure or due to large volume of fragments that are left without retrieval after fragmentation. 28 patients required extracorporeal shockwave lithotripsy as during the procedure these patients had proximal stone migration.
\end{abstract}

Results: The results were analyzed on aspects of stone clearance, hospital stay, complications and patient compliance. 80 patients in Group I, 82 patients in Group II and 42 patients in Group III completed the procedure in single session. The stone clearance at one week for mid ureteric stones in Group I (89.78\%), Group II (91.67\%), and Group III $(91.89 \%$ ) and in lower ureteric stones clearance was in Group I (97.96\%), Group II (96.88\%) and in Group III (95.95\%). At one month $100 \%$ stone clearance was seen in all the three groups. The stented group had more complications than the non stented group.

Conclusion: Ureteroscopic lithotripsy has gained wide acceptance worldwide and is an established technique for lower and mid ureteric calculi when patient demands single session removal of stone and alleviation of symptoms. Routine placement of DJ stent should be discouraged which has more complications as compared to non-stented patients.
Volume 3 Issue 2 - 2016

\author{
Krishna Reddy SV,' Ahammad Basha Shaik ${ }^{2}$ \\ 'Department of Urology, Narayana Medical College, India
}

${ }^{2}$ Department of Statistics, Sri Venkateswara University, India

Correspondence: SV Krishna Reddy, Department of Urology, Narayana Medical College \& Hospital, Nellore-52400I, Andhra Pradesh, India, Tel +9186|2321828, Tel +919849048222, Fax $+9186 \mid 2300068$,

Email krishnareddy@narayanagroup.com

Received: November 04, 2015 | Published: March 05, 2016

Keywords: ureteric calculi, ureteroscopic lithotripsy, complications

Abbreviations: SWL, shock wave lithotripsy; URSL, ureteroscopic lithotripsy; BMI, body mass index; USG, ultrasonography; IVU, intravenous urography; PL, pneumatic lithotripsy; USL, ultrasonic lithotripsy; EHL, eletrohydraulic lithotripsy

\section{Introduction}

Urinary stone disease is a major health problem that concerns millions of patient's worldwide affecting $2-3 \%$ of the human population with a high recurrence rate of almost $50 \%$. Ureteric colic is a urological emergency in terms of the severe pain experienced by the patient. They occur most commonly in men aged between 30years and 60years. ${ }^{1,2}$ It was in 1977 that Goodman reported the first rigid ureteroscopy for therapeutic purposes. ${ }^{3}$ Various treatment modalities have been proposed in literature for ureteral calculi including shock wave lithotripsy (SWL), percutaneous removal, ureteroscopic lithotripsy (URSL), retro-peritoneoscopic ureterolithotomy, laparoscopic ureterolithotomy, and classical open ureterolithotomy., The recent development of small calibre semi rigid and flexible deflectable ureteroscopes and the new generation of various lithotripters have made this procedure safer and more effective. ${ }^{6,7}$
Most of the ureteric stones pass spontaneously and do not require intervention. Spontaneous passage depends on the stone size, shape, location, and associated ureteral edema, which is likely to depend on the length of time that a stone has not progressed.

Management depends upon the stone size, composition, site (location), number, duration, clinical factors of the patient, the availability of the expertise and technology, the cost of the treatment and patient preference. ${ }^{4}$ Placement of ureteric stent after ureteroscopy with stone extraction is usually done to decrease secondary pain caused by mucosal oedema or to prevent possible stenosis. The benchmark for contemporary RIRS using flexible ureterorenoscope and Holmium laser for treatment of renal stones was set by Grasso \& Chalik in $1998 .{ }^{8}$ As this gadgetry is expensive and has high maintenance cost, this is limited to few centres and hence rigid to semi rigid ureteroscopy is still the procedure that is followed at many centres and also in our centre. As symptoms of haematuria, dysuria, and urinary frequency cannot be ignored, hence the need for stenting after uncomplicated ureteroscopic lithotripsy was studied and patients were divided into patients with or with no stenting and patients stented due to intra operative complications. 


\section{Materials and methods}

The medical records of 268 patients who underwent ureterorenoscopic lithotripsy (URSL) by a single surgeon from January 2010 to December 2013 were reviewed retrospectively. Our study was approved by our institutional ethics committee. Written informed consent was taken from all patients for photographing, recording and also its use for scientific and medical education purposes. The consent included the possibility of open surgery if complication occurs and possibility of second endoscopic procedure. All patients were divided into 3 groups. Group I $(\mathrm{n}=98)$ patients had no DJ stent due to small volume calculus and short duration of procedure, while Group II $(n=96)$ patients had DJ stent placed due to large volume calculus and long duration of the procedure but had no fragments that are left for retrieval after fragmentation and stent placement was done with an aim to decrease secondary pain caused by mucosal oedema. Group III $(n=74)$ patients had DJ stent placed due to complications during the procedure or due to large volume of fragments that are left without retrieval after fragmentation.

28 patients in Group III required extracorporeal shockwave lithotripsy for stone clearance as during the ureteroscopic lithotripsy procedure these patients had proximal stone migration. (Table 1) compares the demographic profile of all the 3 groups. The three groups were comparable in terms of Body Mass Index (BMI), stone laterality, number of stones, opacity, stone burden and the preoperative hydronephrosis. Patients presenting with mid or lower ureteric stones of $10 \mathrm{~mm}$ to $22 \mathrm{~mm}$ size, above 18years of age and of either gender were included in this study. Patients with pyonephrosis, severe sepsis and pregnancy were excluded. In patients who had ureteroscopic lithotripsy procedure; whether stented or non-stented the patients were analyzed and results compiled over 268 patients. All the patients' records including history, physical examination, investigations and treatment were reviewed and recorded. Among investigations abdominal ultrasonography (USG), intravenous urography (IVU), CT-abdomen and radioisotope scan, which ever was necessary, was done preoperatively.

Additionally, an abdominal radiograph was obtained in the morning of the day of surgery to know the exact preoperative stone status. Group I comprised of non-stinted patients and Group II \& Group III comprised of stented patients. Pre-operative antibiotics were given to make urine sterile before ureteroscopy. Patients were placed in lithotomy position under spinal anesthesia. Procedure starts with a cystourethroscopy, guide wire insertion (terumo) beyond the stone if possible, ureteroscopy and visualization of stone. Ureteroscopy was done with a semi rigid, offset, tapered ureteroscope (6-7.5 Fr) Karl Storz, Germany) with 5F working channel. Lithotripsy was carried out using pneumatic intracorporeal lithotripter. Multiple transmitted shocks were given and whole stone was fragmented into small particles upto $2 \mathrm{~mm}$ in size. If procedural time was prolonged due large volume of calculus or if retrieval of fragments was not complete after stone disintegration and in all patients with intra operative complications like mmucosal Injury, ureteric Perforation were noted DJ Stent was inserted and these patients were included in Group II \& III respectively. DJ Stent was kept for 1-2weeks in stented group. Patients were discharged within 24-48hours after assessing the postoperative status. Follow up was after 1 week and one month was done. Assessment with USG abdomen and plain X-ray for KUB was done and stone clearance was recorded.

\section{Statistical analysis}

The data values were entered into MS-Excel and statistical analysis has been done by using SPSS Version 20.0 (SPSS Institute Inc., USA). For categorical variables, the values are expressed as number and percentages and to test association between the three groups, the chi-square test was used. For continuous variables, the values are expressed as mean \pm standard deviation and to test mean difference between the three groups, the ANOVA test was used. All p-values are having less than 0.05 are considered as statistically significant.

\section{Results}

Out of 268 patients, 156 (58.21\%) were males, while 112 $(41.79 \%)$ were females. Age range was $18-59$ years with mean age of $37.55 \pm 12.31$ years. Table 1 shows the three groups which were comparable in terms of Body Mass Index (BMI), stone laterality, number of stones, opacity, stone burden and the preoperative hydronephrosis. The stone clearance at 24 hours post-operative period in Group I (79\%), Group II (83\%) Group III (81\%) in mid ureteric and $83 \%, 89.06 \%$ and $84.3 \%$ in lower ureteric stones. At one week stone clearance was in Group I (89.78\%), Group II (91.67\%), and Group III $(91.89 \%)$ for mid ureteric stones and in lower ureteric stone was in Group I (97.96\%), Group II (96.88\%) and in Group III (95.95\%). At one month $100 \%$ stone clearance was seen in all the three groups which were checked with ultrasonography and x-ray KUB. There was no gross difference in stone clearance in three groups. $9(9.2 \%)$ patients in Group I, 8(8.3\%) patients in Group II and 4(8.7\%) in Group III required two sessions for clearance. $28(37.83 \%)$ patients in Group III required extracorporeal shockwave lithotripsy for stone clearance as during the ureteroscopic lithotripsy procedure these patients had proximal stone migration.

The stented group had more complications and patients of this group had irritative bladder symptoms. $26(15.29 \%)$ of these stented patients had severe bladder spasm, steinstrasse and required immediate stent removal at $7^{\text {th }}$ day follow up visit. The complications are shown in Table 2. 98\% patients of this study were discharged from hospital within 24-48hours. Unless retrieval of fragments was not complete after stone disintegration, or had proximal stone migration and in all patients with intra operative complications, our assessment does not support the idea of routine DJ Stent placement in every patient. DJ Stenting although decreases secondary pain caused by mucosal oedema and provides unobstructed urine flow from that particular kidney. $26.5 \%$ of stented group had colic, $10.6 \%$ haematuria, $15.3 \%$ bladder spasm and irritative voiding symptoms and even double chances of developing steinstrasse as compared to non-stinted group (Table 2) (Figure 1).

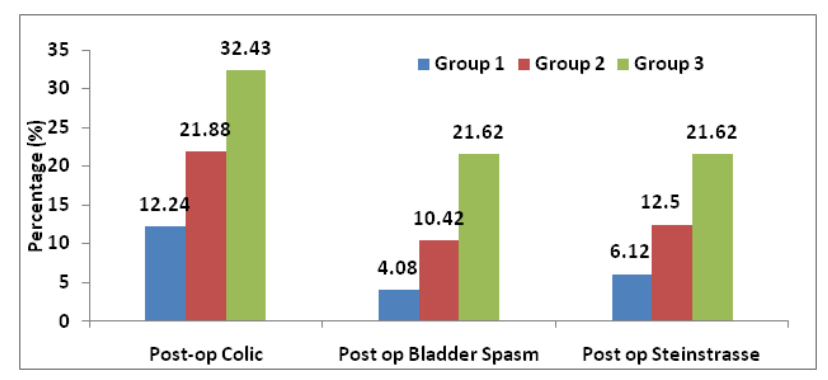

Figure I Comparison of post-op colic, bladder spasm and steinstrasse in all three groups. 
Table I Demographic profile of patients in the three groups

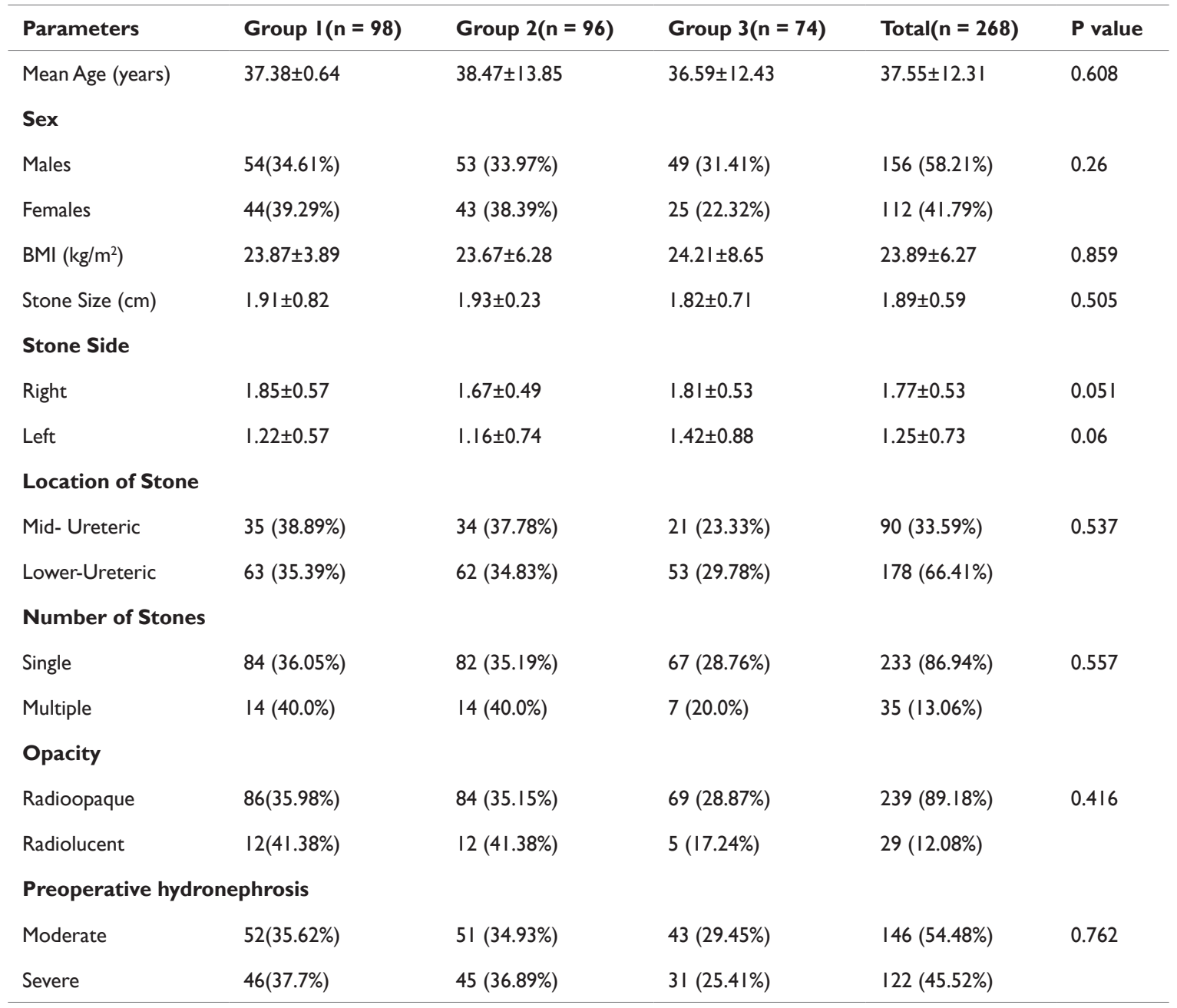

Table 2 Results and complications in all the three groups

\begin{tabular}{|c|c|c|c|c|}
\hline Parameters & Group I (n = 98) & Group 2(n = 96) & Group-3(n= 74) & $P$ value \\
\hline Mean Operative Time (Minutes) & $43.48 \pm 20.83$ & $48.67 \pm 22.32$ & $46.73 \pm 22.17$ & 0.247 \\
\hline Second Look operation & $9(9.18 \%)$ & $8(8.33 \%)$ & $4(5.4 \mid \%)$ & 0.643 \\
\hline ESWL as Second Procedure & $0(0 \%)$ & $0(0 \%)$ & $28(37.84 \%)$ & NA \\
\hline Haematuria & $6(6.12 \%)$ & $12(12.5 \%)$ & $16(21.62 \%)$ & $0.01 *$ \\
\hline Mucosal Injury & $0(0 \%)$ & $0(0 \%)$ & $2(2.7 \%)$ & 0.07 \\
\hline Ureteric Perforation & $0(0 \%)$ & $0(0 \%)$ & $2(2.7 \%)$ & 0.07 \\
\hline Post-op Colic & $12(12.24 \%)$ & $21(21.88 \%)$ & $24(32.43 \%)$ & $0.006 *$ \\
\hline Post op Bladder Spasm & $4(4.08 \%)$ & $10(10.42 \%)$ & $16(21.62 \%)$ & $0.001 *$ \\
\hline Post-op Steinstrasse & $6(6.12 \%)$ & $12(12.5 \%)$ & $16(21.62 \%)$ & $0.01 *$ \\
\hline Post Operative Fever & $9(9.18 \%)$ & II (II.46\%) & $14(18.92 \%)$ & 0.149 \\
\hline \multicolumn{5}{|l|}{ Stone clearance at I week } \\
\hline Mid Ureteric & 87 (89.78\%) & 88 (91.67\%) & 68 (91.89\%) & 0.886 \\
\hline Lower Ureteric & $96(97.96 \%)$ & $93(96.88 \%)$ & $71(95.95 \%)$ & 0.736 \\
\hline Extravasation & $0(0 \%)$ & $0(0 \%)$ & I (I.35\%) & 0.268 \\
\hline Hospital Stay (Days) & $1.12 \pm 0.58$ & $\mathrm{I} .14 \pm 0.75$ & $1.35 \pm 0.66$ & 0.055 \\
\hline
\end{tabular}

$*_{\mathrm{p}}<0.05$, significant; NA, not applicable 


\section{Discussion}

Ureteroscopic lithotripsy has become the method of choice for the management of distal ureteric calculi in many centers worldwide, although some prefer SWL. Although SWL is of a non-invasive nature, nowadays some authors recommend URSL for distal ureteral calculi as a first-line treatment since it provides higher success rates and quick stone clearance with minimal complications. ${ }^{10,11}$ Factors affecting safety and effectiveness of intraureteral fragmentation of stones by endoscopic techniques include: stone size, its location and length of time within the ureter, infection and urinary retention, type of instrument used and the operator's experience. With the miniaturization of the ureteroscope and use of small caliber intracorporeal lithotripsy devices, the complication and pain associated with ureteroscopy have decreased with time. ${ }^{9}$

The main advantage of URSL is because it offers both immediate reliefs from symptoms and good stone fragmentation. SWL may be an alternative treatment option, however, this method may be initially unsuccessful in $10 \%$ to $30 \%$ of patients, requiring multiple sessions, and taking a lengthly for stone-free status. ${ }^{10-12}$ Ureteroscopy is usually favoured when stones are larger than $10 \mathrm{~mm}$, are known to be cystine, appear radiolucent or possibly occur in women of childbearing age. ${ }^{10-12}$ We worked only with a semi-rigid ureteroscope, and the stones were fragmented with a pneumatic lithotripter. The success rate was attributable to the learning curve and accumulation of experience with ureteroscopy, the availability of small rigid and semi rigid ureteroscopes and the pneumatic lithotripter that are highly efficient and economical instruments for stone fragmentation. Review of published series from the last decade on treatment with ureteroscopy using a variety of ureteroscopes and intracorporeal lithotripsy devices reveals success rates ranging from $86 \%$ to $100 \%{ }^{12,13}$

In our material, we obtained a success rate at a level of $90.9 \%$ after a single URSL procedure. This is consistent with published results reported by other centers performing pneumatic lithotripsy. The highest, almost $100 \%$, effectiveness was obtained in the case of stones smaller than $10 \mathrm{~mm}$ in diameter, located within the lower ureter. Effectiveness decreased alongside with the increase of the stone diameter, its location within the upper sections of the ureter, longer period within the ureter, and the presence of urinary retention. With different energy levels used, URS is classified into pneumatic lithotripsy (PL), ultrasonic lithotripsy (USL) and electrohydraulic lithotripsy (EHL). Of these, PL, developed in the 1990s, was reported to be the most effective, ${ }^{13,14}$ therefore, it was employed in this study. The pneumatic lithotripter is simple and works with compressed air, providing good results at a lower cost. ${ }^{15}$ But it has some disadvantages. The main disadvantages of pneumatic lithotripsy are that it produces larger fragments that potentially which may cause more problems in term of spontaneous passage ${ }^{16}$ or retropulsion of larger fragments during the procedure. ${ }^{17}$

Teichman et al., ${ }^{16}$ demonstrated that out of all methods available it is the holmium laser that breaks up stones into the smallest fragments. However, lasers are costly and are not available everywhere. Therefore, some authors recommend using baskets or graspers to remove larger fragments produced by the pneumatic lithotripter to reduce complications and also reduce the second procedure. ${ }^{17-19}$ As we also used stone forceps in most of the procedures the second procedure was not performed except in only few of our patients. However, use of stone forceps caused mucosal injury in two patients $(1.8 \%)$ that was treated with DJ stenting for 3 weeks. Previous reports in China documented the incidence of complications of the lower ureteral calculi to be $2 \% \sim 8 \%,{ }^{20}$ mainly including injury to the ureter, such as perforation, laceration, hematuria and ureteral stricture, and all of these are liable to occur with unskillful practice. In our study, a DJ stent was placed for 3 7days in patients in Group II who underwent Ureteroscopic lithotripsy, as some authors have advised that the placement of DJ stent effectively prevents postoperative infection and helps drainage of urine and fragmented stones after the operation. ${ }^{21}$

Moreover, if ureteral perforation or laceration is found, the DJ stent is needed to be placed for 4weeks and for most of time; such management is usually successful and requires no open repair. ${ }^{22}$ In our study also we had $2(1.8 \%)$ cases of ureteric perforation and were managed by placement of stent for 4 weeks. Avulsion of ureter is the most serious complication of ureteroscopic procedure if ever occurs. ${ }^{23,24}$ Most of these complications can be managed conservatively except avulsion. Mucosal injury observed in different studies range from $3.5-5 \%,{ }^{18,24}$ and mucosal injury in our study was seen in $2(1.8 \%)$ patients. Some studies declared procedural failure or repeat procedure in range of $3-13 \%{ }^{25,26}$ In our study we had a repeat procedure rate of $7.8 \%$ which is the same as it has been reported in literature. Cevik et al. ${ }^{27}$ evaluated the necessity of leaving the ureteral stent.

They did not recommend doing this routinely after a complicationfree URSL. The AUA ureteral stones clinical guidelines panel and EAU guidelines on urolithiasis have reported that stenting following uncomplicated ureteroscopy is optional. ${ }^{28} \mathrm{~A}$ meta-analysis published by the EAU-AUA Guidelines panel has evaluated the most relevant complications of ureteroscopy - sepsis, steinstrasse, stricture, ureteric injury and urinary tract infection. ${ }^{29}$ The overall complication rates reported in recent literature are $5-9 \%$, with a $1 \%$ rate of significant complications. Serious complications, including renal loss and death, were rare..$^{29}$ A standardized complication reporting methodology is necessary to enable appropriate comparisons between institutions, time periods, or innovations in technique. ${ }^{30,31}$

The issue remains whether the higher efficiency quotient for URSL (97\%) compared with ESWL (58\%) is the sole or primary consideration for choice of treatment option..$^{32}$ Its calculation takes both the rate of retreatment and the rate of secondary intervention into consideration. It is not worthy, however, efficiency is emerging as a more and more important parameter in the cost containment in current health care provision. There are in fact many patients who will opt for the more definitive (URSL) rather than the least invasive (ESWL) technique, especially if they were responsible for the cost of treatment. The treatment options to be offered should therefore be unbiased and as practical as possible in relation to the merits and limitations in a particular setting and the patient's preference.

\section{Conclusion}

Ureteroscopic lithotripsy has gained wide acceptance worldwide and is an established technique. Lithotripsy using Pneumatic Litho last is perhaps the cheapest and quite efficient technique in managing ureteric stones intracorporeally. Routine placement of stents after URSL should be avoided except in complicated cases and large volume of fragments stone burden.

\section{Acknowledgements}

None.

\section{Conflict of interest}

Author declares that there is no conflict of interest. 


\section{References}

1. Romero V, Akpinar H, Assimos DG. Kidney stones: A global picture of prevalence, incidence, and associated risk factors. Rev Urol. 2010;12(2-3):e86-e96.

2. Papadoukakis S, Stolzenburg J, Truss MC. Treatment strategies of ureteral stones. EAU-EBU Update Series. 2006;4:184-190.

3. Basillote JB, Lee DI, Eichel L, et al. Ureteroscopes: flexible, rigid, and semirigid. Urol Clin N Am. 2004;31(1):21-32.

4. Gettman MT, Segura JW. Management of ureteric stones: issues and controversies. BJU Int. 2005;95(Suppl 2):85-93.

5. el-Faqih SR, Husain I, Ekman PE, et al. Primary choice of intervention for distal ureteric stone: ureteroscopy or ESWL? $\mathrm{Br} \mathrm{J} \mathrm{Urol.}$ 1988;62(1):13-18.

6. Marberger M, Hofbauer J, Turk C, et al. Management of ureteric stones. Eur Urol. 1994;25:265-272.

7. Preminger GM. Technique versus technology. What is the most appropriate method for removal of ureteral calculi. J Urol. 1994;152:66-67.

8. Grasso M, Chalik Y. Principles and applications of laser lithotripsy: experience with the holmium laser lithotrite. J Clin Laser Med Surg. 1998;16(1):3-7.

9. Dretler SP, Cho G. Semirigid ureteroscopy: A new genre. J Urol. 1989;141:1314-1318.

10. Peschel R, Janetschek G, Bartsch G. Extracorporeal shock wave lithotripsy versus ureteroscopy for distal ureteral calculi: a prospective randomized study. J Urol. 1999;162(6):1909-1912.

11. Honeck P, Häcker A, Alken P, et al. Shock wave lithotripsy versus ureteroscopy for distal ureteral calculi: a prospective study. Urol Res. 2006;34(3):190-192.

12. Turk TM, Jenkins AD. A comparison of ureteroscopy to in situ extracorporeal shock wave lithotripsy for the treatment of distal ureteral calculi. J Urol. 1999;161(1):45-46.

13. Küpeli B, Biri H, Isen K, et al. Treatment of ureteral stones: comparison of extracorporeal shock wave lithotripsy and endourologic alternatives. Eur Urol. 1998;34(6):474-479.

14. Biri H, Kupeli B, Isen K, et al. Treatment of lower ureteral stones: extracorporeal shockwave lithotripsy or intracorporeal lithotripsy? $J$ Endourol. 1999;13(2):77-81.

15. Yagisawa T, Kobayashi C, Ishikawa N, et al. Benefits of ureteroscopic pneumatic lithotripsy for the treatment of impacted ureteral stones. $J$ Endourol. 2001;15(7):697-699.
16. Teichman JM, Vassar GJ, Bishoff JT, et al. Holmium YAG lithotripsy yields smaller fragments than lithoclast, pulsed dye laser or electrohydraulic lithotripsy. J Urol. 1998;159(1):17-23.

17. Mitre AI, Chambo Arap S. Ureteroscopy. Glenn's Urologic Surgery. Philadelphia: Lippincott-Raven Publishers; 1998. p. 942-945.

18. Hamano S, Nomura H, Kinsui H, et al. Experience with Ureteral Stone Management in 1,082 Patients Using Semirigid Ureteroscopes. Urol Int. 2000;65:106-111

19. Aridogan IA, Zeren S, Bayazit Y, et al. Complications of Pneumatic Ureterolithotripsy in the Early Postoperative Period. J Endourology. 2005;19(1):50-53.

20. Wu KJ, Li X. Endourology for treatment of upper urinary tract calculi: A ten-year summary. Chin J Endourol \& ESWL. 1995;1:62-63.

21. Lingeman JE, Woods JR, Nelson DR. Commentary on ESWL and blood pressure. J Urol. 1995;154(1):2-4.

22. Sun YH, Wang LH, Qian SX. Treatment of urinary stones with pneumatic ureteroscopic lithotripsy. Chin J Urol. 1999;20:2-4.

23. Benyamin JC, Donaldson PJ, Hill JT. Ureteric Perforation after Ureteroscopy-Conservative management. Urology. 1987;29(6):623-624.

24. Carter SS, C Cox R, Wickham JE. Complications associated with Ureteroscopy. Br J Urol. 1986;58(6):625-628.

25. Srivastava A, Gupta R, Kumar A, et al. Routine stenting after ureteroscopy for distal ureteral calculi is unnecessary: results of a randomized controlled trial. J Endourol. 2003;17(10):871-874.

26. El Nehas AR, El-Tabey NA, Eraky I, et al. Semirigid Ureteroscopy for Ureteral stone: A multivariate analysis of Unfavourable Results. J Urol. 2009;181(3):1158-1162.

27. Cevik I, Dilloglugil O, Akdas A, et al. Is stent placement necessary after uncomplicated ureteroscopy for removal of impacted ureteral stones? $J$ Endourol. 2010;24:1263-1267.

28. Preminger GM, Tiselius HG, Assimos DG, et al. guideline for the management of ureteral calculi. Eur Urol. 2007;52(6):1610-1631.

29. Tiselius HG, Alken P. Guidelines on Urolithiasis 2009. European Association of Urology. 2008.

30. Krishna Reddy SV, Shaik AB, Sreenivas K. Penile injuries: A 10 year Experience. Can Urol Assoc J. 2014;8(9-10):626-631.

31. Dindo D, Demartines N, Clavien PA. Classification of surgical complications: A new proposal with evaluation in a cohort of 6336 patients and results of a survey. Ann Surg. 2004;240(2):205-213.

32. Preminger GM, Tiselius HG, Assimos DG, et al. 2007 guideline for the management of ureteral calculi. J Urol. 2007;178(6):2418-2434. 